\title{
COMPARATIVE EFFECTIVENESS OF BIPHOSPHONATES AND PARATHYROIDECTOMY IN OSTEOPOROSIS RELATED TO PRIMARY HYPERPARATHYROIDISM
}

\section{J. Cárdenas, B. Barquiel, C. Alvarez-Escola, B. Lecumberri, B. Pelegrina, L.F. Pallardo, A. Torrijos Endocrinology and Nutrition Department. "La Paz" University Hospital. Madrid}

\section{INTRODUCTION}

The use of Bisphosphonates could be a treatment option of Osteoporosis secondary to Primary Hyperparathyroidism (OPHP) in patients not eligible or that refuse/reject surgery.

\section{METHODS/DESIGN}

We analyzed data of 46 patients diagnosed of OPHP. Fourteen underwent surgery and 32 were treated with Bisphosphonates at standard dose. Densitometric parameters and of calcium-phosphorus metabolism were analyzed before and after 1 and 2 years of treatment. The analysis was performed with SPSS-20.0.

\section{RESULTS}

Table 1. Baseline characteristics of patients with primary hyperparathyroidism

\begin{tabular}{|l|c|c|c|} 
& $\begin{array}{c}\text { Bisphosphonate } \\
\text { s treatment } \\
\text { group }\end{array}$ & $\begin{array}{c}\text { Surgery } \\
\text { treatment } \\
\text { group }\end{array}$ & Pa value \\
\hline $\mathrm{N}$ & 32 & 14 & \\
\hline Age (years) & $65 \pm 8$ & $61 \pm 11$ & 0,178 \\
\hline Body mass index (kg/m2) & $26.2 \pm 4.9$ & $25.0 \pm 4.4$ & 0,441 \\
\hline Menopause age (years) & $47 \pm 6$ & $50 \pm 6$ & 0,192 \\
\hline Years since menopause & $18 \pm 9$ & $13 \pm 9$ & 0,159 \\
\hline $\begin{array}{l}\text { Smoking habit }(\geq 10 \\
\text { cigarettes per day) }\end{array}$ & $8(57.1)$ & $4(12.9)$ & 0,002 \\
\hline $\begin{array}{l}\text { Alcohol consumption }(\geq 3 \\
\text { units per day) }\end{array}$ & 0 & $1(2.3 \%)$ & 0,295 \\
\hline $\begin{array}{l}\text { Consumption of calcium } \\
\text { ( } \geq 3 \text { glasses of milk or } \\
\text { equivalent) }\end{array}$ & $7(87.5)$ & $19(73.1)$ & 0,645 \\
\hline Creatinine clearance & $81 \pm 30$ & $88 \pm 31$ & 0,526 \\
\hline $\begin{array}{l}\text { Previous low trauma } \\
\text { fracture }\end{array}$ & $9(28.1)$ & $1(7.1)$ & 0,112 \\
\hline Vitamine D & $\begin{array}{c}22.4 \\
(12.5-28.3)\end{array}$ & $\begin{array}{c}16.1 \\
(8.5-23.75)\end{array}$ & 0.203 \\
\hline
\end{tabular}
Data as $\mathrm{N}(\%)$, Mean \pm Standard Deviation or Median (Interquartile Range). Significance of differences among pairs of groups if $p<0.05$.

There were not differences in age, BMI, menopause age, years since menopause, alcohol consumption, diagnosis of previous low-trauma fracture, calcium intake, creatinine clearance, serum PTH, urinary calcium, serum alkaline phosphatase, urinary D-pyridinoline, vitamine $D$ neither in the mineral density (MD) of Lumbar spine, femoral neck or tota hip at baseline between groups. Smoking was more frequent and serum calcium lower in the bisphosphonate group (BG) $(p<0,05)$. (Table 1) Serum calcium and PTH decreased after 1 and 2 years of treatment within surgery group $(S G)(p<0,01)$ but remained stable in the $B G$. Urinary calcium decreased in the SG, urine D-pyridinoline decreased in the BG and Serum alkaline phosphatase decreased in both groups after 1 and 2 years. There were no differences between groups. (Table 2) The lumbar spine mineral density (MD) increased after 1 year $(0.754 \pm 0.121$ vs $0.785 \pm 0.132 ; p<0,01)$ and 2 years $(0.754 \pm 0.121$ vs $0.791 \pm 0.107 ; p<0.01)$ in the BG, and only after 2 years $(0.749 \pm 0.144$ vs $0.794 \pm 0.189 ; p<0,05)$ in the SG. Femoral neck MD increased after 1 year $(0.622 \pm 0.091$ vs $0.640 \pm 0.093 ; p<0,01)$ but remained stable after 2 years in the BG. It also remained stable in the $S G$ and in total hip $M D$ in both groups. No differences were found between groups in MD in any location. (Table 3)

\begin{tabular}{|c|c|c|c|c|}
\hline & $\begin{array}{l}\text { Years of } \\
\text { treatment }\end{array}$ & $\begin{array}{l}\text { Bisphosphonates } \\
\text { treatment group }\end{array}$ & $\begin{array}{l}\text { Surgery treatment } \\
\text { group }\end{array}$ & Pa value \\
\hline Serum calcium (mg/dl) & $\begin{array}{l}\text { Baseline } \\
\text { 1-year } \\
\text { 2-years }\end{array}$ & $\begin{array}{l}10.2 \pm 0.7 \\
10.1 \pm 0.7 \\
10.2 \pm 0.6\end{array}$ & $\begin{array}{r}10.8 \pm 0.9 \\
9.6 \pm 0.4^{* *} \\
9.5 \pm 0.4^{* *}\end{array}$ & $\begin{array}{l}0.028^{*} \\
0.011^{*} \\
0.001^{* *}\end{array}$ \\
\hline Serum PTH (ng/l) & $\begin{array}{l}\text { Baseline } \\
\text { 1-year } \\
\text { 2-years }\end{array}$ & $\begin{array}{l}121.0 \pm 35.2 \\
111.4 \pm 47.1 \\
120.1 \pm 59.1\end{array}$ & $\begin{array}{l}141.3 \pm 68.9 \\
68.8 \pm 26.5^{\star *} \\
57.5 \pm 34.0^{* *}\end{array}$ & $\begin{array}{l}0,324 \\
0.050^{*} \\
0.001^{* *}\end{array}$ \\
\hline Urine calcium (mg/dl) & $\begin{array}{l}\text { Baseline } \\
\text { 1-year } \\
\text { 2-years }\end{array}$ & $\begin{array}{l}12.6(7.2-18.2) \\
11.9(8.1-15.8) \\
11.8(8.7-17.8)\end{array}$ & $\begin{array}{c}16.6(7.4-25.6) \\
9.7(5.8-13.0)^{*} \\
10.4(5.7-18.6)^{*}\end{array}$ & $\begin{array}{l}0,346 \\
0,297 \\
0,666\end{array}$ \\
\hline Serum alkaline phospatase & $\begin{array}{l}\text { Baseline } \\
\text { 1-year } \\
\text { 2-years }\end{array}$ & $\begin{array}{l}110.0(71.8-181.4) \\
67.0(55.8-94.2)^{\star *} \\
69.5(57.0-88.8)^{\star *}\end{array}$ & $\begin{array}{l}98.9(71.8-130.0) \\
74.0(64.0-88.0)^{\star *} \\
70.0(57.5-91.0)^{* *}\end{array}$ & $\begin{array}{l}0,531 \\
0,546 \\
0,844\end{array}$ \\
\hline Urine D-pyridinoline & $\begin{array}{l}\text { Baseline } \\
1 \text {-year } \\
\text { 2-years }\end{array}$ & $\begin{array}{l}8.9(6.8-12.3) \\
7.2(5.4-8.1)^{\star *} \\
5.9(4.5-7.1)^{\star *}\end{array}$ & $\begin{array}{l}8.0(6.4-10.2) \\
6.5(4.6-7.4) \\
6.9(3.5-9.2)\end{array}$ & $\begin{array}{l}0,589 \\
0,355 \\
0,507\end{array}$ \\
\hline
\end{tabular}

Data as $\mathrm{N}(\%)$. Mean \pm Standard Deviation or Median (Interquartile Range). ${ }^{*}, \mathrm{P}<0.050 ;{ }^{*}, \mathrm{P}<0.010$ by comparison to baseline. ${ }^{a}$ Significance of differences among pairs of groups if $\mathrm{P}<0.05$.

Table 3. Bone mineral density changes in patients with primary hyperparathyroidism after medical or surgical treatment

Years of Bisphosphonates

Surgery treatment treatment group group value

Lumbar spine mineral density $(\mathrm{g} / \mathrm{cm} 2)$

$\begin{array}{lccc}\text { Baseline } & 0.754 \pm 0.121 & 0.749 \pm 0.144 & 0,899 \\ \text { 1-year } & 0.785 \pm 0.132^{* *} & 0.797 \pm 0.179 & 0,827 \\ \text { 2-years } & 0.791 \pm 0.107^{* *} & 0.794 \pm 0.189^{*} & 0,955 \\ \text { Baseline } & -3.04 \pm 1.09 & -2.99 \pm 1.34 & 0,905 \\ \text { 1-year } & -2.68 \pm 1.08^{* *} & -2.67 \pm 1.48 & 0,982 \\ \text { 2-years } & -2.62 \pm 1.00^{* *} & -2.51 \pm 1.71^{*} & 0,809 \\ & & & \\ \text { Baseline } & 0.622 \pm 0.091 & 0.606 \pm 0.088 & 0,588 \\ \text { 1-year } & 0.640 \pm 0.093^{* *} & 0.599 \pm 0.107 & 0,262 \\ \text { 2-years } & 0.632 \pm 0.097 & 0.609 \pm 0.109 & 0,526 \\ \text { Baseline } & -2.58 \pm 0.97 & -2.59 \pm 0.93 & 0,971 \\ \text { 1-year } & -2.41 \pm 0.95^{* *} & -2.70 \pm 0.95 & 0,415 \\ \text { 2-years } & -2.46 \pm 1.05 & -2.69 \pm 1.08 & 0,546 \\ \text { Baseline } & 0.737 \pm 0.105 & 0.672 \pm 0.165 & 0,323 \\ \text { 1-year } & 0.752 \pm 0.104 & 0.670 \pm 0.149 & 0,083 \\ \text { 2-years } & 0.706 \pm 0.132 & 0.702 \pm 0.139 & 0,929 \\ \text { Baseline } & -1.50(2.70-1.10) & -1.55(4.20-1.20) & 0,524 \\ \text { 1-year } & -1.90(2.4-1.2) & -1.90(3.5-1.3) & 0,407 \\ \text { 2-years } & -2.00(2.68-1.48) & -2.00(2.93-1.43) & 0,605\end{array}$

Femoral neck mineral density $(\mathrm{g} / \mathrm{cm} 2)$

Femoral neck T score (SD)

$* \mathrm{P}<0.050 \cdot * *, \mathrm{P}<0.010$ by comparison to baseline

ata as Mean \pm Standard Deviation or Median (Interquartile Ranc

asignificance of differences among pairs of groups if $P<0.05$.

\section{CONCLUSIONS}

Bisphosphonates treatment could be as effective as parathyroidectomy in the treatment of OPHP

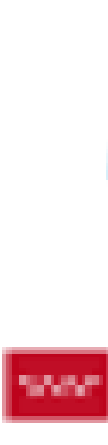

\section{References}

Khan AA, Bilezikian JP, Kung AW et al. Alendronate in primary
hyperparathyroidism: a double-blind, randomized, placebo-controlled trial. J Clin Endocrinol Metab. 2004 Jul;89(7):3319-25.

Dy BM, Grant CS, Wermers RA et al. Changes in bone mineral density after surgical intervention for primary hyperparathyroidism.. Surgery. 2012 Dec;152(6):1051-8. 